\title{
Renewal and memory origin of anomalous diffusion: A discussion of their joint action
}

\author{
Mauro Bologna, ${ }^{1, *}$ Bruce J. West, ${ }^{2, \dagger}$ and Paolo Grigolini ${ }^{3, \dagger}$ \\ ${ }^{1}$ Instituto de Alta Investigación, Universidad de Tarapacá-Casilla 6-D Arica, Chile \\ ${ }^{2}$ Information Science Directorate, Army Research Office, Research Triangle Park, North Carolina 27709, USA \\ ${ }^{3}$ Center for Nonlinear Science, University of North Texas, P.O. Box 311427, Denton, Texas 76203-1427, USA
}

(Received 10 August 2013; published 2 December 2013)

\begin{abstract}
The adoption of the formalism of fractional calculus is an elegant way to simulate either subdiffusion or superdiffusion from within a renewal perspective where the occurrence of an event at a given time $t$ does not have any memory of the events occurring at earlier times. We illustrate a physical model to assign infinite memory to renewal anomalous diffusion and we find (i) a condition where the simultaneous action of a renewal and a memory source of subdiffusion generates localization and (ii) a condition where they make subdiffusion weaker and superdiffusion emerge. We argue that our approach may provide important contributions to the current search to distinguish the renewal from the memory source of subdiffusion.
\end{abstract}

DOI: 10.1103/PhysRevE.88.062106

PACS number(s): 02.50.-r, 05.40.Fb

\section{INTRODUCTION}

Strange kinetics [1,2] constitute the underlying dynamics of subdiffusive and superdiffusive processes that appear to be ubiquitous in physical [3], social [4], and biological [5] phenomena. The term strange was adopted from chaotic dynamical systems theory as an application of the notion of fractal space and time to explain the microscopic dynamics of anomalous transport [1] in complex phenomena. In strange kinetics the familiar integer differential formalism is replaced with the fractional differential formalism in order to adequately describe the dynamics of superdiffusion and subdiffusion. In the phase space equations describing the evolution of a probability density function (PDF) the fractional formalism implies that the statistics are renewal. In fact, subdiffusion can be simulated by replacing discrete random walk processes with the continuous time random walk processes of Montroll and Weiss [6]. On the other hand superdiffusion can be generated by assuming that at any time step in the random walk the statistics of the walker's jumps has a diverging second moment [4]. Both cases satisfy the renewal condition because the waiting times and the jump intensities are randomly selected from distributions with a diverging first moment and a diverging second moment, respectively, with no correlation between drawings.

Given the increasing level of activity in the field of strange kinetics it is virtually impossible to provide an authoritative bibliography and so we restrict our attention here to addressing a single technical issue. That issue concerns the proper way to incorporate memory into the fractional diffusion equation for the PDF describing an anomalous diffusion process. The observed experimental results are reproduced using the fractional diffusion equation $[3,4]$

$$
\frac{\partial^{\alpha}}{\partial t^{\alpha}} p(x, t)=D \frac{\partial^{\beta}}{\partial x^{\beta}} p(x, t), \quad p(x, t=0)=\delta(x), \quad t \geqslant 0
$$

\footnotetext{
*mauroh69@ gmail.com

†bruce.j.west.civ@mail.mil

${ }^{\ddagger}$ grigo@unt.edu
}

with $\alpha \leqslant 1$ and $\beta \leqslant 2$. The condition $\alpha=1, \beta=2$ corresponds to the ordinary diffusion equation, which only applies in simple media. Real diffusion processes in complex media are called anomalous since they obey the more general fractional diffusion equations with $\alpha \neq 1$ and/or $\beta \neq 2$. We use the Caputo fractional derivatives in time defined in terms of the Laplace transform as

$$
\mathcal{L} \mathcal{T}\left\{\frac{\partial^{\alpha}}{\partial t^{\alpha}} p(x, t) ; s\right\}=s^{\alpha} \widehat{p}(x, s)-s^{\alpha-1} p(x, 0),
$$

where $\widehat{p}(x, s)$ denotes the Laplace transform of the PDF in time. One way to construct the fractional derivative in time is by assuming that the random walker spends a very large amount of time at each site, with a waiting time PDF

$$
\psi(\tau) \propto 1 / \tau^{1+\alpha} .
$$

See Ref. [7] for recent work on the issues connecting a fractional derivative in time with underlying stochastic processes. We adopt the Riesz fractional derivative in space defined in terms of the Fourier transform in the symmetric case to be

$$
\mathcal{F} \mathcal{T}\left[\frac{\partial^{\beta}}{\partial x^{\beta}} p(x, t) ; k\right]=-|k|^{\beta} \widetilde{p}(k, t),
$$

where $\widetilde{p}(k, t)$ is the Fourier transform of the PDF in space and is known as the characteristic function. We refer the readers to the excellent review paper [4] and to the original paper [8] for discussions of Eq. (4).

To make the present paper as self-contained as possible we devote Sec. II to a concise derivation of the stochastic Liouville equation method, which we use to generate infinite memory in the conventional case and which is manifest in a timedependent diffusion coefficient. In this case the waiting-time PDF has a finite first moment, the PDF of jump lengths has a finite second moment, and the scaling index of the solution to the diffusion equation involves the scaling indices from both space and time. The more general situation including diverging average waiting times and/or infinite second spatial moments is discussed in Sec. III. The exact solution to the fractional diffusion equation in space and time along with infinite memory, produced through an inverse power-law bath 
spectrum, is obtained in this section and shown to satisfy the generic scaling equation

$$
p(x, t)=\frac{1}{t^{\eta}} F\left(\frac{x}{t^{\eta}}\right) .
$$

In Sec. IV we study the $\alpha<1$ diffusion process for a particle under the influence of bath fluctuations generated by the subordination to fluctuations already possessing an infinite memory. In Sec. V we draw some conclusions.

\section{DYNAMICAL ORIGIN OF INFINITE MEMORY}

The dynamics of mechanical systems are Hamiltonian based and the equations of motion can be expressed in terms of the Liouville operator. We adopt the Liouville operator technique here and use it to construct a Fokker-Planck equation for the PDF when the thermal bath is non-Ohmic, which is to say the thermal fluctuations are correlated in time with an inverse power-law autocorrelation function. It is shown that under these conditions the Liouvillian is itself a stochastic operator.

\section{A. Stochastic Liouville equation}

We begin our discussion with the familiar case of a diffusing particle characterized by the dynamic variable $X(t)$. Assume that the dynamics of the particle can be described by

$$
\frac{d X(t)}{d t}=\xi(t),
$$

where the driving force is the sum over the environmental variables

$$
\xi(t)=\sum_{j} \xi_{j}(t) .
$$

The set of variables $\left\{\xi_{j}\right\}$ denotes the degrees of freedom of the environment which we model here as a heat bath with which the diffusing particle is in contact. In the latter case the bath variable would be an oscillator with frequency $\omega_{j}$. In principle we should study the Liouville equation for the coupled system

$$
\frac{\partial}{\partial t} p_{T}\left(x, \xi_{1}, \ldots, \xi_{j}, \ldots ; t\right)=L p_{T}\left(x, \xi_{1}, \ldots, \xi_{j}, \ldots ; t\right)
$$

from which we can derive the equation of motion for the reduced PDF where $p(x, t) d x$ is the probability that the dynamic variable $X(t)$ lies in the interval $(x, x+d x)$ at time $t$ :

$$
p(x ; t)=\int d \xi_{1} \cdots d \xi_{j} \cdots p_{T}\left(x, \xi_{1}, \ldots, \xi_{j}, \ldots ; t\right) .
$$

In order to carry out the coarse graining indicated in Eq. (9) we partition the Liouville operator into two parts

$$
L=L_{1}+L_{B}
$$

with the bath-particle coupling determined by

$$
L_{1} \equiv-\xi \frac{\partial}{\partial x}
$$

and $L_{B}$ denotes the Liouvillian for a set of harmonic oscillators constituting the heat bath. The equation of motion for the reduced distribution density $p(x, t)$ can be obtained using the Zwanzig projection method $[9,10]$. However, for the sake of simplicity we herein adopt the stochastic Liouville equation method [11], which reads

$$
\frac{\partial}{\partial t} \sigma(x, t)=L_{1}(t) \sigma(x, t),
$$

to describe the evolution of the stochastic PDF $\sigma(x, t)$.

The intuitive foundation of this useful equation is based on the adoption of the interaction picture corresponding to Eq. (9). In this picture the interaction Liouvillian becomes time dependent and reads

$$
L_{1}(t)=-\xi(t) \frac{\partial}{\partial x} .
$$

The formal solution of Eq. (12) is given by the operator equation

$$
\sigma(x, t)=\exp \left(\int_{0}^{t} L_{1}\left(t^{\prime}\right) d t^{\prime}\right) \sigma(x, 0) .
$$

It is important to notice that because $\xi(t)$ is a random variable the PDF $\sigma(x, t)$ determined by Eq. (14) is also a stochastic quantity. Consequently, an average over many realizations of $\xi(t)$ has to be made to establish the desired PDF $p(x, t)$ :

$$
p(x, t)=\left\langle\exp \left(\int_{0}^{t} L_{1}\left(t^{\prime}\right) d t^{\prime}\right)\right\rangle p(x, 0),
$$

since

$$
p(x, t)=\langle\sigma(x, t)\rangle \text { and } p(x, 0)=\sigma(x, 0) .
$$

The central limit theorem applied to the bath requires that the statistics of $\xi(t)$ be Gaussian, which enables us to evaluate the average in Eq. (15) to obtain

$$
p(x, t)=\exp \left[\frac{1}{2}\left\langle\left(\int_{0}^{t} \xi\left(t^{\prime}\right) d t^{\prime}\right)^{2}\right) \frac{\partial^{2}}{\partial x^{2}}\right] p(x, 0)
$$

whose time derivative generates the following diffusion equation:

$$
\frac{\partial}{\partial t} p(x, t)=\frac{1}{2} D(t) \frac{\partial^{2}}{\partial x^{2}} p(x, t) .
$$

The time-dependent diffusion coefficient in Eq. (18) is given by the integral over the bath autocorrelation function

$$
D(t) \equiv 2\left\langle\xi^{2}\right\rangle_{\mathrm{eq}} \int_{0}^{t} \Phi_{\xi}(\tau) d \tau,
$$

where the bath autocorrelation function is defined in terms of the average over the equilibrium bath

$$
\Phi_{\xi}(t)=\frac{\langle\xi(0) \xi(t)\rangle_{\mathrm{eq}}}{\left\langle\xi^{2}\right\rangle_{\mathrm{eq}}} .
$$

On the other hand, the time integration of Eq. (6) yields

$$
X(t)=\int_{0}^{t} d t^{\prime} \xi\left(t^{\prime}\right)+X(0) .
$$

Setting the initial condition $X(0)=0$ and again assuming that $\xi(t)$ is a stationary Gaussian variable with vanishing mean value we obtain

$$
\left\langle X(t)^{2}\right\rangle=2\left\langle\xi^{2}\right\rangle_{\mathrm{eq}} \int_{0}^{t} d t^{\prime} \int_{0}^{t^{\prime}} \Phi_{\xi}(\tau) d \tau .
$$


The time rate of change of the second moment of the dynamic variable

$$
\frac{d\left\langle X(t)^{2}\right\rangle}{d t}=2\left\langle\xi^{2}\right\rangle_{\mathrm{eq}} \int_{0}^{t} \Phi_{\xi}(\tau) d \tau=D(t)
$$

which therefore provides a second way to calculate the timedependent diffusion coefficient.

\section{B. Non-Ohmic bath as a source of time-dependent diffusion coefficients}

It is interesting to note that the time dependence of the diffusion coefficient is a direct consequence of the structure of the bath autocorrelation function, which in turn stems from the bath spectrum. Let us assume that each oscillator of the bath system generating the environmental fluctuation $\xi(t)$ has a specific frequency $\omega$. The autocorrelation function $\Phi_{\xi}(t)$ can then be expressed, using the Wiener-Khintchin theorem, as

$$
\Phi_{\xi}(t)=\int_{0}^{\infty} d \omega \rho(\omega) \cos (\omega t) .
$$

We assume that the frequency distribution $\rho(\omega)$ has the powerlaw form

$$
\rho(\omega) \propto \omega^{\delta-1},
$$

to obtain

$$
\Phi_{\xi}(t)=\frac{\int_{0}^{\infty} d \omega \omega^{\delta-1} \cos (\omega t)}{\int_{0}^{\infty} d \omega \omega^{\delta-1}} .
$$

We determine the scaling behavior of the autocorrelation function by transforming to the integration variable $z=\omega t$ and using simple algebra to factor the time from the integral in Eq. (26):

$$
\Phi_{\xi}(t) \propto \frac{\operatorname{sgn}(1-\delta)}{t^{\delta}} .
$$

The well-known Tauberian theorem is

$$
\mathcal{L} \mathcal{T}\left\{\frac{1}{t^{\psi}}\right\}=\left(\frac{1}{s}\right)^{1-\psi} \Gamma(1-\psi),
$$

where as noted in the Introduction $\mathcal{L} \mathcal{T}\{f(t)\}$ denotes the Laplace transform of $f(t)$, and can be used to obtain for the Laplace transform of $\Phi_{\xi}(t)$ in Eq. (27), denoted by $\hat{\Phi}_{\xi}(s)$ :

$$
\hat{\Phi}_{\xi}(s) \propto s^{\delta-1} .
$$

Consequently, the Laplace transform of the diffusion coefficient denoted by $\hat{D}(s)$ using Eq. (23) becomes

$$
\hat{D}(s) \propto \frac{1}{s^{2-\delta}} .
$$

Inverse Laplace transforming this expression and again using the Tauberian theorem we obtain

$$
D(t) \propto \frac{1}{t^{\delta-1}} .
$$

The inverse power-law behavior of the diffusion coefficient is a direct consequence of the assumed power-law spectrum of the bath oscillators.

\section{Connection between non-Ohmic bath and Hurst coefficient}

The Hurst coefficient $H$ denotes the scaling behavior of the diffusing particle in anomalous diffusion. This means that the second moment $\left\langle X^{2}(t)\right\rangle$ is expected to have the asymptotic property

$$
\left\langle X^{2}(t)\right\rangle \propto t^{2 H} .
$$

On the other hand, according to Eq. (22), the second derivative of the second moment yields the autocorrelation function

$$
\frac{d^{2}}{d t^{2}}\left\langle X^{2}(t)\right\rangle \propto \Phi_{\xi}(t) .
$$

Equating the coefficients for the scaling of the autocorrelation function in Eqs. (27) and (33) we obtain

$$
H=1-\frac{\delta}{2} \text {. }
$$

As a consequence of this relation the time-dependent diffusion coefficient $D(t)$ in Eq. (31) can be rewritten in terms of the Hurst exponent as

$$
D(t) \propto t^{2 H-1}
$$

This analysis leads us to express the diffusion equation (18) as

$$
\frac{\partial}{\partial t} p(x, t)=D_{H} t^{2 H-1} \frac{\partial^{2}}{\partial x^{2}} p(x, t)
$$

with $0<H<1$. It is important to stress that earlier work [12] has proven that Eq. (36) leads to results equivalent to the well-known theory of fractional Brownian motion (FBM) [13].

On the basis of these theoretical arguments we propose to include memory into the anomalous renewal process described by Eq. (1) by means of the following prescription:

$$
\frac{\partial^{\alpha}}{\partial t^{\alpha}} p(x, t)=D_{H} t^{2 H-1} \frac{\partial^{\beta}}{\partial x^{\beta}} p(x, t) .
$$

The main result of this paper is to obtain the exact solution to Eq. (37), but before we get to that let us examine the scaling behavior of some related dynamic equations.

\section{Scaling}

Equation (37) is an important equation whose general solution illustrated in this paper recovers the interesting results discussed in the recent review paper [14]. To address this problem, it is convenient to study Eq. (36) first. Let us express Eq. (36) in the Fourier representation to obtain the dynamic equation for the characteristic function, the Fourier transform of the PDF:

$$
\frac{\partial}{\partial t} \tilde{p}(k, t)=-k^{2} D_{H} t^{2 H-1} \tilde{p}(k, t)
$$

The time integration of this equation yields

$$
\tilde{p}(k, t)=\exp \left[-k^{2} \frac{D_{H} t^{2 H}}{2 H}\right]
$$

since the characteristic function has the initial condition $\tilde{p}(k, 0)=1$. Here we introduce dimensional analysis and adopt the notation that $[y]$ denotes the dimension of the variable $y$. The scaling of the characteristic function is determined by the condition that the dynamic variable scales as

$$
[x]=[t]^{\eta}
$$


together with the product in the exponential $k^{2} t^{2 H}$ being kept constant such that

$$
[k]=[t]^{-H} \text {. }
$$

Since the phase space and Fourier variables are inversely related $[x]=1 /[k]$ the two scaling exponents of the time must be equal,

$$
\eta=H .
$$

Now consider the more complicated condition of a diffusion equation with a fractional spatial derivative:

$$
\frac{\partial}{\partial t} p(x, t)=D_{H} t^{2 H-1} \frac{\partial^{\beta}}{\partial x^{\beta}} p(x, t) .
$$

The adoption of the Fourier transform in this case and using Eq. (4) yields [8]

$$
\frac{\partial}{\partial t} \widetilde{p}(k, t)=-|k|^{\beta} D_{H} t^{2 H-1} \widetilde{p}(k, t),
$$

which simply integrates to

$$
\widetilde{p}(k, t)=\exp \left[-|k|^{\beta} \frac{D_{H} t^{2 H}}{2 H}\right] .
$$

Using analogous dimensional arguments to those adopted to obtain Eq. (42) yields for the relation between scaling exponents

$$
\eta=\frac{2 H}{\beta} .
$$

The authors of the recent work [7] have shown that moving from the integer partial differential operator in time in Eq. (43) to that of the fractional differential operator in time in Eq. (37) corresponds to the time dilatation

$$
[t]=[\tau]^{1 / \alpha} .
$$

To implement this relation we interpret the time in Eq. (43) as the operational time $\tau$, thereby interpreting the scaling of Eq. (46) as

$$
[x]=[\tau]^{2 H / \beta} .
$$

From Eq. (47) we therefore require the equality between chronological time $t$ and operational time $\tau$ to be

$$
[\tau]=[t]^{\alpha},
$$

and inserting Eq. (49) into Eq. (48) we obtain for the dimensional scaling in terms of chronological time

$$
[x]=[t]^{\eta},
$$

with the relation between scaling exponents

$$
\eta=\frac{2 H \alpha}{\beta} \text {. }
$$

The scaling result given by Eq. (51) is found to be incorrect, and the theory subsequently developed herein explains how to obtain the correct scaling. Using Eq. (43) we are led to the following equality between the dimensions:

$$
\frac{1}{[t]^{\alpha}}=\frac{[t]^{2 H-1}}{[x]^{\beta}}=\frac{[t]^{2 H-1}}{[t]^{\beta \eta}},
$$

which equating coefficients immediately yields

$$
\eta=\frac{\alpha+2 H-1}{\beta} \text {. }
$$

Since $\eta<0$ does not make sense physically, because it would imply the regression to the origin of a spreading PDF, we conclude that $\alpha+2 H-1<0$ implies $\eta=0$ and consequently would be a localization process due to the joint action of two different sources of subdiffusion: one renewal $(\alpha<1)$ and one implying infinite memory $(H<0.5)$.

It is interesting to notice that when $\alpha=1$, this prediction recovers the result of Eq. (46). This last result coincides with the one recently proposed by Eliazar and Shlesinger [14].

\section{JOINT ACTION OF TWO SOURCES OF SUBDIFFUSION}

In this section we illustrate a way to solve Eq. (37), the central equation of this article, and we show using this solution that in fact the correct prediction for the scaling $\eta$ is given by Eq. (53).

\section{A. General solution of Eq. (37)}

Consider the following fractional diffusion equation with infinite memory:

$$
\frac{\partial^{\alpha}}{\partial t^{\alpha}} P(x, t)=D t^{2 H-1} \frac{\partial^{\beta}}{\partial x^{\beta}} P(x, t) .
$$

To solve Eq. (54) first eliminate the spatial derivative by taking the spatial Fourier transform and using the Riesz derivative $[4,8]$ obtain the fractional dynamic equation for the characteristic function

$$
\frac{\partial^{\alpha}}{\partial t^{\alpha}} \widetilde{P}(k, t)=-D t^{2 H-1}|k|^{\beta} \widetilde{P}(k, t) .
$$

We assume that the characteristic function $\widetilde{P}(k, t)$ is analytic and can be expressed in the series form

$$
\widetilde{P}(k, t)=\sum_{n=0}^{\infty} c_{n}(k) t^{n \gamma} .
$$

Insert Eq. (56) into Eq. (55)and using the Caputo derivative [15] in time yields

$\sum_{n=1}^{\infty} c_{n}(k) \frac{\Gamma(n \gamma+1)}{\Gamma(n \gamma+1-\alpha)} t^{n \gamma-\alpha-2 H+1}=-D|k|^{\beta} \sum_{n=0}^{\infty} c_{n}(k) t^{n \gamma}$.

Selecting the scaling exponent in the series expansion to be $\gamma=\alpha+2 H-1$ we obtain

$\sum_{n=0}^{\infty} c_{n+1}(k) \frac{\Gamma[(n+1) \gamma+1]}{\Gamma[(n+1) \gamma+1-\alpha]} t^{n \gamma}=-D|k|^{\beta} \sum_{n=0}^{\infty} c_{n}(k) t^{n \gamma}$.

The above equation gives the recurrence relationship for the expansion coefficients

$$
c_{n+1}(k)=-D|k|^{\beta} \frac{\Gamma[(n+1) \gamma+1-\alpha]}{\Gamma[(n+1) \gamma+1]} c_{n}(k),
$$




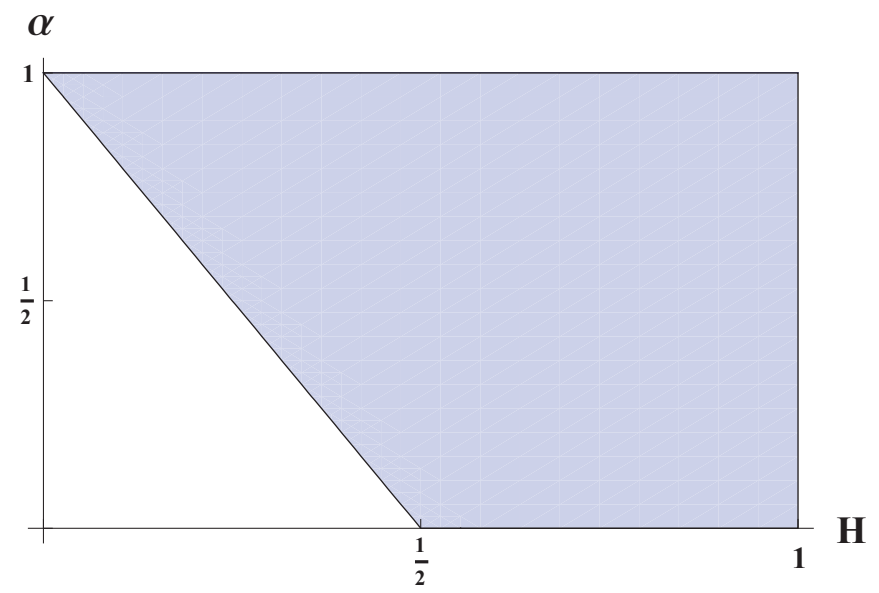

FIG. 1. (Color online) Region of parameter values allowed by Eq. (61).

which implies

$c_{n}(k)=c_{0}\left(-D|k|^{\beta}\right)^{n} \prod_{i=0}^{n-1} \frac{\Gamma[(i+1) \gamma+1-\alpha]}{\Gamma[(i+1) \gamma+1]}, \quad n \geqslant 1$.

In order for the series solution to converge the exponent must satisfy $\gamma \geqslant 0$ implying the condition we imposed earlier on physical grounds,

$$
\gamma=\alpha+2 H-1 \geqslant 0
$$

The plot of the corresponding allowed region of the parameter values, for $0 \leqslant \alpha \leqslant 1$ and $0 \leqslant H \leqslant 1$, is depicted in Fig. 1 . It is rather straightforward to demonstrate that the radius of convergence of the solution is infinite.

Since we are looking for a normalized $P(x, t)$ we require $\widetilde{P}(0, t)=1$. Using this normalization condition the solution of Eq. (55) can be written as follows:

$$
\widetilde{P}(k, t)=\frac{1}{\Gamma[1-\alpha]} \sum_{n=0}^{\infty}\left(-|k|^{\beta} t^{\gamma} D\right)^{n} \prod_{i=0}^{n} \frac{\Gamma[i \gamma+1-\alpha]}{\Gamma[i \gamma+1]}
$$

and consequently the PDF solution to the fractional phase space equation with memory is given by the inverse Fourier transform

$$
\begin{aligned}
P(x, t)= & \int_{-\infty}^{\infty} \frac{d k}{2 \pi} \frac{\exp [-\imath k x]}{\Gamma[1-\alpha]} \sum_{n=0}^{\infty}\left(-|k|^{\beta} t^{\gamma} D\right)^{n} \\
& \times \prod_{i=0}^{n} \frac{\Gamma[i \gamma+1-\alpha]}{\Gamma[i \gamma+1]} .
\end{aligned}
$$

Note that $P(x, t)$ given by Eq. (63) is of the form

$$
P(x, t)=\frac{1}{\phi(t)} F\left[\frac{x}{\phi(t)}\right], \quad \text { with } \quad \phi(t)=t^{\gamma / \beta}
$$

as expected. Asymptotically, for $x \rightarrow \infty$ and $\beta<2$, we have the inverse power law

$$
\begin{aligned}
P(x, t) & \approx D \frac{\Gamma[1-\alpha+\gamma]}{\Gamma[1+\gamma]} \frac{\Gamma[1+\beta] \sin \left[\frac{\pi \beta}{2}\right]}{\pi} \frac{t^{\gamma}}{|x|^{1+\beta}} \\
& =D \frac{\Gamma[2 H]}{\Gamma[\alpha+2 H]} \frac{\Gamma[1+\beta] \sin \left[\frac{\pi \beta}{2}\right]}{\pi} \frac{t^{\alpha+2 H-1}}{|x|^{1+\beta}} .
\end{aligned}
$$

It is important to notice that this general solution fits the typical scaling prescription of diffusion processes,

$$
P(x, t)=\frac{1}{t^{\eta}} F\left(\frac{x}{t^{\eta}}\right)
$$

with the scaling index $\eta$ given by the prediction in Eq. (53).

\section{B. Particular cases}

Consider the particular case of the solution given by Eq. (62) with the parameter values $\alpha=1$ (or $\gamma=2 H$ ). It follows that

$$
\frac{1}{\Gamma[1-\alpha]} \prod_{i=0}^{n} \frac{\Gamma[i \gamma+1-\alpha]}{\Gamma[i \gamma+1]}=\frac{1}{n ! \gamma^{n}}
$$

and the series for the characteristic function becomes

$$
\widetilde{P}(k, t)=\sum_{n=0}^{\infty} \frac{\left(-|k|^{\beta} t^{\gamma} D\right)^{n}}{n ! \gamma^{n}}=\exp \left[-|k|^{\beta} \frac{D}{2 H} t^{2 H}\right] .
$$

Consequently the PDF in this case is the Lévy distribution obtained by taking the inverse Fourier transform and $\beta$ is the Lévy index.

Consider a second particular case with the parameter value $2 H=1($ or $\gamma=\alpha)$. Then we have

$$
\frac{1}{\Gamma[1-\alpha]} \prod_{i=0}^{n} \frac{\Gamma[i \gamma+1-\alpha]}{\Gamma[i \gamma+1]}=\frac{1}{\Gamma[n \gamma+1]},
$$

and the characteristic function becomes

$$
\widetilde{P}(k, t)=\sum_{n=0}^{\infty} \frac{\left(-|k|^{\beta} t^{\gamma} D\right)^{n}}{\Gamma[n \gamma+1]}=E_{\alpha}\left[-|k|^{\beta} D t^{\alpha}\right],
$$

where $E_{\alpha}[z]$ is the Mittag-Leffler function (MLF). Note that since the MLF is a stretched exponential at early times that the Fourier transform of the characteristic function (70) at early times is again a Lévy PDF but with a different time dependence than that given by Eq. (68).

\section{MOVING THE BATH FROM OPERATIONAL TO CHRONOLOGICAL TIME}

A recent dynamical interpretation of the Caputo fractional time derivative [7] corresponds to replacing the operational time experienced by a complex phenomenon with the chronological time measured by the clock on the wall. Therefore it is interesting to study the situation in which we interpret the dynamics of the bath oscillators as taking place in operational time. We then shift our perspective from the operational to the chronological time. For each of the oscillators, according to the new theoretical perspective [7] the harmonic time evolution $\cos (\omega \tau)$ is expressed in terms of the Mittag-Leffler function 
$E_{\alpha}\left(i \omega t^{\alpha}\right)$. For this reason we have to adopt the procedure illustrated below in Sec. IV A to establish the special form of the time-dependent diffusion coefficient $D(t)$ obtained in this case.

\section{A. Evaluation of the bath autocorrelation function}

We study the transition from operational to chronological time by using the Weiner-Khinchin theorem in the two representations for the bath autocorrelation function:

$$
\begin{aligned}
\Phi_{\xi}(\tau) & =\int_{0}^{\infty} \rho_{\xi}(\omega) \cos \omega \tau d \omega \\
& \Rightarrow \operatorname{Re}\left[\int_{0}^{\infty} \rho_{\xi}(\omega) E_{\alpha}\left(i \omega t^{\alpha}\right) d \omega\right] .
\end{aligned}
$$

The bath autocorrelation function can be evaluated by taking the Laplace transform to obtain

$$
\begin{gathered}
\mathcal{L} \mathcal{T}\left\{\operatorname{Re}\left[\int_{0}^{\infty} \rho_{\xi}(\omega) E_{\alpha}\left(i \omega t^{\alpha}\right) d \omega\right] ; s\right\} \\
=\int_{0}^{\infty} \omega^{\delta-1} \frac{s^{2 \alpha-1}}{\omega^{2}+s^{2 \alpha}} d \omega=\frac{\pi s^{\delta \alpha-1}}{2 \sin \frac{\pi}{2} \delta},
\end{gathered}
$$

where we have again used the power-law spectrum for the bath. Inverting the Laplace transform of the bath autocorrelation function and employing the Tauberian theorem from Eq. (28) we finally obtain

$$
\operatorname{Re}\left[\int_{0}^{\infty} \rho(\omega) E_{\alpha}\left(i \omega t^{\alpha}\right) d \omega\right]=\frac{\pi}{2 \sin \frac{\pi}{2} \delta} \frac{1}{\Gamma[1-\delta \alpha] t^{\delta \alpha}} .
$$

Consequently in this case the time-dependent diffusion coefficient is

$$
D(t) \propto 1 / t^{\delta \alpha-1} .
$$

The scaling index for the diffusion coefficient is here a consequence of the correlated bath fluctuations in operational time and reduces to the earlier result for $\alpha=1$.

\section{B. The new diffusion equation}

The scaling parameter $\delta$ in Eq. (74) is related to the Hurst exponent by Eq. (34):

$$
\delta=2(1-H)
$$

Thus, in the case when the bath oscillators evolving operational time memory is included in the diffusion process

$$
\frac{\partial^{\alpha}}{\partial t^{\alpha}} p(x, t)=D_{\alpha, H} t^{1-\alpha \delta} \frac{\partial^{\beta}}{\partial x^{\beta}} p(x, t),
$$

then by means of the new scaling indices we obtain

$$
\frac{\partial^{\alpha}}{\partial t^{\alpha}} p(x, t)=D_{\alpha, H} t^{1-2 \alpha(1-H)} \frac{\partial^{\beta}}{\partial x^{\beta}} p(x, t) .
$$

Using the same dimensional procedure as that adopted to obtain the scaling index in Eq. (53) yields in the present situation

$$
\eta=\frac{1+\alpha(2 H-1)}{\beta}
$$

It is interesting to rewrite this scaling index in the form

$$
\eta=\frac{2 \alpha H}{\beta}+\Delta \eta
$$

where the shift from the previously obtained index is given by

$$
\Delta \eta=\frac{1-\alpha}{\beta}
$$

Equation (79) vindicates our assertion that Eq. (51) is not the correct scaling relation. The reason that Eq. (51) is not the correct scaling is because it corresponds to realizing the scaling $2 H / \beta$ when the dynamics are unfolding in operational time. Consequently, the result of this operational dynamics is transferred with no change but the time dilatation between two consecutive values of the phase space variable in operational time, to that in the chronological time representation. However, the result of Eq. (73) shows that the autocorrelation function $\Phi_{\xi}(t) \propto 1 / t^{\delta}$, moving from operational to chronological time, becomes $\Phi_{\xi}(t) \propto 1 / t^{\alpha \delta}$. This may generate a significant effect. The subdiffusional condition $\delta>1$ can turn into the superdiffusional condition $\alpha \delta<1$, for $\alpha$ sufficiently small. This is the reason why the scaling $\eta$ is always larger than the scaling coefficient predicted by Eq. (51).

\section{CONCLUDING REMARKS}

We emphasize that the order in which the fractional derivative and the long-term memory are obtained from the bath dynamics is important for obtaining the proper scaling. The scaling index found in the general case differs from that obtained by Eliazar and Shlesinger [14] based on the indices for the Joseph effect (divergence in time) and the Noah effect (divergence in space) and obtained by completely different arguments. The results of the present article afford an extension of their work [14] to the case where $\alpha<1$. This is a timely issue, since in the literature there is active research into strange kinetics that establishes the origin of anomalous diffusion in biological processes [16] and proposals are being made to assess if the origin of subdiffusion is renewal or implies the infinite memory of FBM [17].

The results of the present analysis may prove useful in a number of research areas. For example, a recent study of the processes of random growth of surfaces [18] has adopted the renewal assumption and a diffusion theory equivalent to Eq. (37) with $\alpha<1, H=0.5$, and $\beta=2$. More recent research has revisited this earlier work using the infinite memory of FBM [19], namely, a diffusion equation equivalent to Eq. (37) with $\alpha=1, H<0.5$, and $\beta=2$, and found that the persistence properties emerging from the numerical treatment are reproduced more properly than in the earlier work. Another area of application is in the analysis of brain dynamics [20] adopting the hypothesis that the brain undergoes phase transition. This theoretical perspective leads to the conjecture that criticality generates renewal events and with them $1 / f$ noise, without ruling out the possibility of a joint action of renewal and memory [21]. The single column of a surface growing, thanks to the experimental procedure of molecular epitaxy [18], under specific conditions of cooperation with the other units, is expected to show signs of criticality and with it renewal events properly described by the 
Mittag-Leffler function. However, slight changes in the growth model may generate FBM memory, as recently proven [22]. It is plausible that conditions exist where the diffusion processes are generated by the joint action of renewal and memory, and the results of this article will facilitate further progress on these issues.

\section{ACKNOWLEDGMENTS}

M.B. acknowledges financial support from FONDECYT Project No. 1110231. P.G. warmly thanks ARO and Welch for their support through Grants No. W911NF-11-1-0478 and No. B-1577, respectively.
[1] M. F. Shlesinger, G. M. Zaslavsky, and J. Klafter, Nature (London) 363, 31 (1993).

[2] M. Bologna, P. Grigolini, and B. J. West, Chem. Phys. 284, 115 (2002).

[3] C. Ingo, R. L. Magin, L. Colon-Perez, W. Triplett, and T. H. Mareci, Magn. Reson. Med., doi: 10.1002/mrm.24706.

[4] A. A. Dubkov, B. Spagnolo, and V. V. Uchaikin, Int. J. Bifurcation Chaos Appl. Sci. Eng. 18, 2649 (2008).

[5] F. Höfling and T. Franosch, Rep. Prog. Phys. 76, 046602 (2013).

[6] E. W. Montroll and G. H. Weiss, J. Math. Phys. 6, 167 (1965).

[7] P. Pramukkul, A. Svenkeson, P. Grigolini, M. Bologna, and B. J. West, Adv. Math. Phys. 2013, 498789 (2013).

[8] B. J. West and V. Seshadri, Physica A 113, 203 (1982).

[9] R. Zwanzig, in Quantum Statistical Mechanics, edited by P. H. E. Meijer (Gordon-Breach, New York, 1966), pp. 139-172.

[10] P. Grigolini, Adv. Chem. Phys. 62, 1 (1986).

[11] R. Kubo, J. Math. Phys. 4, 174 (1963).

[12] R. Cakir, P. Grigolini, and A. A. Krokhin, Phys. Rev. E 74, 021108 (2006).
[13] B. B. Mandelbrot and J. W. van Ness, SIAM Rev. 10, 422 (1968).

[14] I. I. Eliazar and M. F. Shlesinger, Phys. Rep. 527, 101 (2013).

[15] F. Mainardi and R. Gorenflo, J. Comput. Appl. Math. 118, 283 (2000).

[16] E. Barkai, Y. Garini, and R. Metzler, Phys. Today 65(8), 29 (2012).

[17] Y. Meroz, I. M. Sokolov, and J. Klafter, Phys. Rev. Lett. 110, 090601 (2013).

[18] R. Failla, P. Grigolini, M. Ignaccolo, and A. Schwettmann, Phys. Rev. E 70, 010101 (2004).

[19] E. Geneston, M. Bologna, A. Krokhin, and P. Grigolini, Phy. Rev. E (to be published).

[20] P. Allegrini, D. Menicucci, R. Bedini, L. Fronzoni, A. Gemignani, P. Grigolini, B. J. West, and P. Paradisi, Phys. Rev. E 80, 061914 (2009).

[21] M. Zare and P. Grigolini, Phys. Rev. E 86, 051918 (2012).

[22] A. Taloni, A. Chechkin, and J. Klafter, Phys. Rev. Lett. 104, 160602 (2010). 